IUHET-473

\title{
Gauge Invariance and the Pauli-Villars Regulator in Lorentz- and CPT-Violating Electrodynamics
}

\author{
B. Altschul ${ }^{1}$ \\ Department of Physics \\ Indiana University \\ Bloomington, IN 47405 USA
}

\begin{abstract}
We examine the nonperturbative structure of the radiatively induced Chern-Simons term in a Lorentz- and CPT-violating modification of QED. Although the coefficient of the induced Chern-Simons term is in general undetermined, the nonperturbative theory appears to generate a definite value. However, the CPT-even radiative corrections in this same formulation of the theory generally break gauge invariance. We show that gauge invariance may yet be preserved through the use of a Pauli-Villars regulator, and, contrary to earlier expectations, this regulator does not necessarily give rise to a vanishing ChernSimons term. Instead, two possible values of the Chern-Simons coefficient are allowed, one zero and one nonzero. This formulation of the theory therefore allows the coefficient to vanish naturally, in agreement with experimental observations.
\end{abstract}

\footnotetext{
${ }^{1}$ baltschu@indiana.edu
} 
One of the most interesting terms that arises in the study of Lorentz- and CPTviolating corrections to the standard model action [1, 2, 3] is the electromagnetic ChernSimons term, with Lagrange density $\mathcal{L}_{C S}=\frac{1}{2}\left(k_{C S}\right)_{\mu} \epsilon^{\mu \alpha \beta \gamma} F_{\alpha \beta} A_{\gamma}$,4, 5, 6]. $\mathcal{L}_{C S}$ is not gauge invariant; it changes by a total derivative under a gauge transformation. However, the associated action is gauge invariant, and it generates a modified theory in which photons of different polarizations propagate at different speeds. The resulting birefringence has been searched for in the light from distant galaxies [6, 7, 8, but no such effect has been seen. This constrains $k_{C S}$ to effectively vanish.

It is interesting to ask whether the Chern-Simons term may be generated as a radiative correction and, if so, whether the physical term's effectively vanishing value can be reconciled with the possibility of nonzero Lorentz violation in other sectors of the theory. The relevant Lagrange density for studying these questions is [9]

$$
\mathcal{L}=-\frac{1}{4} F^{\mu \nu} F_{\mu \nu}+\bar{\psi}\left(i \not \partial-m-e \not A-\not \gamma \gamma_{5}\right) \psi
$$

This theory has the potential to induce a finite radiatively-generated Chern-Simons term, with $\Delta k_{C S}$ proportional to $b$. However, the coefficient of proportionality depends upon the regularization [10, 11, 12. If the regulator used enforces the gauge invariance of the induced Lagrange density, then $\Delta k_{C S}$ must necessarily vanish [13]; however, this is not particularly interesting, because it excludes the existence of a Chern-Simons term $a$ priori. Other regulators lead to different values of $\Delta k_{C S}$, and through a suitable choice, any coefficient of proportionality between the two may be found. This ambiguity has been extensively studied, and several potentially interesting values of $\Delta k_{C S}$ have been identified [11, 14, 15, 16, 17, 18. Most notably, if the theory is defined nonperturbatively in $b$, then the ambiguity is lessened, and a single value appears to be preferred [10, 11, 14]. However, since this value is nonzero, there is a potential for conflict with the observed vanishing of $k_{C S}$.

We shall continue the analysis of the nonperturbatively-defined theory. While the induced Chern-Simons term itself has been extensively studied, the higher-order, CPT-even corrections to this theory have largely been neglected. In a nonperturbatively defined theory, the terms of all orders in $b$ are tied together, and so information about the CPT-even terms may help clarify the structure of the CPT-odd Chern-Simons term. We have previously demonstrated [19] that the $\mathcal{O}\left(b^{2}\right)$ terms in the photon self-energy may violate the Ward identity that enforces the transversality of the vacuum polarization $-p_{\mu} \Pi^{\mu \nu}(p)=0$; however, our calculation was limited to the case of $m=0$. We shall demonstrate here that the same result holds in the opposite limit, when $\left|b^{2}\right| \ll m^{2}$ (which represents the physical regime for all electrically charged elementary particles). This failure of transversality could represent a significant problem for the nonperturbative formulation of the theory. However, this difficulty may be overcome through the use of a Pauli-Villars regulator. Introducing such a regulator changes the nature of the ambiguity in the Chern-Simons term. When the Ward-identity-violating terms are eliminated, we are left with a theory 
in which the coefficient $\Delta k_{C S}$ of the induced Chern Simons term is not in any way forced to vanish, yet it still may vanish quite naturally. This may represent a resolution of the physical paradox described above.

We shall calculate the $\mathcal{O}\left(b^{2}\right)$ contribution to the zero-momentum photon self-energy, $\Pi^{\mu \nu}(p=0)$, under the assumption that $\Pi^{\mu \nu}(p)$ may be expanded as a power series in $b$. However, we do not expect this assumption to be generically valid. The exact fermion propagator,

$$
S(l)=\frac{i}{\not-m-\not b \gamma_{5}},
$$

may be rationalized to obtain [11, 14]

$$
S(l)=i \frac{\left(\not+m-\not b \gamma_{5}\right)\left(l^{2}-m^{2}-b^{2}+[\not, \not b] \gamma_{5}\right)}{\left(l^{2}-m^{2}-b^{2}\right)^{2}+4\left[l^{2} b^{2}-(l \cdot b)^{2}\right]} .
$$

At $l=0$, the denominator of the rationalized propagator becomes $\left(m^{2}+b^{2}\right)^{2}$. The square root $\left|m^{2}+b^{2}\right|$ of this expression arises in the calculation of $\Pi^{\mu \nu}(p=0)$, and the absolute value leads to behavior that is nonanalytic in $b$. The $b$-odd portion of the selfenergy (which is just the Chern-Simons term) has different forms for $-b^{2} \leq m^{2} \neq 0$, $-b^{2}<m^{2} \neq 0$, and $m=0$. For the $b$-even terms, we also expect the power-series representation of the self-energy to break down at $b^{2}=-m^{2}$; moreover, there may exist other thresholds for nonanalytic behavior as well. We shall therefore restrict our attention to the regime in which $\left|b^{2}\right| \ll m^{2}$. Since large values of $b^{2}$ are excluded for most physical particles, this is a reasonable restriction.

To control the high-energy behavior of our theory, we shall use a Pauli-Villars regulator. By utilizing this method of regulation, we may deal with the usual divergences that arise in the photon self-energy [at $\left.\mathcal{O}\left(b^{0}\right)\right]$, as well as the Ward-identity-violating terms that appear at $\mathcal{O}\left(b^{2}\right)$. The use of a single regulator at all orders in $b$ is necessary, because in the nonperturbative formalism, there is actually only a single Feynman diagram that contributes to the photon self-energy at $\mathcal{O}\left(e^{2}\right)$. This diagram is the usual QED vacuum polarization, but with the usual fermion propagator replaced by the $b$-exact $S(l)$. We shall use a symmetric integration prescription in our calculation, because this is appropriate at $\mathcal{O}\left(b^{0}\right)$, and it preserves the expected transformation properties of the loop integral. (Moreover, without symmetric integration, additional ambiguities in $\Delta k_{C S}$ would exist.)

The Pauli-Villars regularization of the photon self-energy involves the introduction of a fictitious species of heavy fermions, whose contribution to the self-energy is subtractive. This subtraction renders the Lorentz-invariant part of the self-energy finite and preserves its gauge invariance. Previously, it has been believed that the same subtraction will cause the $\mathcal{O}(b)$ Chern-Simons term to vanish. However, we shall show that this is not necessarily the case.

The fictitious Pauli-Villars particles have the exact propagator

$$
S_{M}(l)=\frac{i}{\not-M-\not \gamma_{M} \gamma_{5}}
$$


where $M$ is the particles' large mass, and $b_{M}$ is the Lorentz-violating coefficient appropriate to these fictitious particles. Previous analyses have assumed that $b_{M}=b$, and indeed this must be the case in a perturbatively defined theory, in which the $b$ interaction is treated as a vertex. However, in the nonperturbative formulation, this restriction is absent, and $b$ and $b_{M}$ exist as distinct objects. Depending upon what properties we demand for the self-energy, $b_{M}$ may take on different values. Even if we place quite strong (yet quite natural) conditions on the form of $\Pi^{\mu \nu}(p)$, we are still left with a discrete freedom in our choice of $b_{M}$; either $b_{M}=b$ or $b_{M}=-b$ will suffice.

In fact, the weakest condition that we may place upon $b_{M}$ is no condition at all. If we choose not to worry about the failure of the Ward identity at $\mathcal{O}\left(b^{2}\right)$, then the only restriction on the Pauli-Villars propagator is that it must cancel the divergences at $\mathcal{O}\left(b^{0}\right)$. In that case, any value of $b_{M}$ is acceptable. We might be tempted to choose a vanishing $b_{M}$, which would produce a formulation of the theory equivalent to that used in [10]. However, since Lorentz violation is already present in the theory, there is no reason to prefer this value. Similarly, the choice $b_{M}=b$, while aesthetically pleasing, has nothing special to recommend it. We are left with an arbitrary $b_{M}$, which will generate an arbitrary Chern-Simons term. Moreover, since $b$ and $b_{M}$ need not point in the same direction, the coefficient $\Delta k_{C S}$ of the induced Chern-Simons term need not even be parallel to $b$; this is an even greater degree or arbitrariness in $\Delta k_{C S}$ than has been found previously.

The above scenario illustrates an interesting point. In the theory as we have described it, there can exist a radiatively-induced Chern-Simons term even if $b$ vanishes, because the regulator involves the Lorentz-violating parameter $b_{M}$. In a general Lorentz-violating quantum field theory, the Lagrange density need not be the only source of the violation. The regularization prescription can also break Lorentz invariance, and the Lorentz violation of the regulator need not be determined by the behavior of any terms in the unrenormalized action. It may seem somewhat unnatural for the regulator to become an additional source of Lorentz violation. However, it is well established that fictitious Pauli-Villars fermions may generate radiative corrections that are qualitatively different from those generated by a theory's real fermions. For example, in massless QED, with a Pauli-Villars regulator, it is the fictitious heavy particles which are solely responsible for the anomalous nonconservation of the axial vector and dilation currents. However, the specific scenario presented in the previous paragraph is probably unrealistic, because we had to abandon the Ward identity in order to avoid placing any restrictions on $b_{M}$.

The key to developing a more sophisticated theory - one in which gauge invariance holds at all orders - is the determination of the $\mathcal{O}\left(b^{2}\right)$ part of the self-energy. Since we are only interested in the situation in which we may expand $\Pi^{\mu \nu}(p)$ as a power series in $b$, we may use the approximation scheme developed in [10] to expand the exact propagator to second order in $b$. We find

$$
S(l) \approx \frac{i}{\not-m}+\frac{i}{\not-m}\left(-i \not b \gamma_{5}\right) \frac{i}{\not-m}+\frac{i}{\not-m}\left(-i \not b \gamma_{5}\right) \frac{i}{\not-m}\left(-i \not b \gamma_{5}\right) \frac{i}{\not-m} .
$$

Naively, this might appear equivalent to treating the $b$ term as an interaction vertex. 
However, there are subtle differences that characterize our nonperturbative approach; since there is only a single diagram, there is a complex interplay between terms at different orders in $b$.

The one-loop photon self-energy is

$$
\Pi^{\mu \nu}(p)=-i e^{2} \int \frac{d^{4} k}{(2 \pi)^{4}} \operatorname{tr}\left\{\gamma^{\mu} S(k) \gamma^{\nu} S(k+p)\right\}+\Pi_{M}^{\mu \nu}(p) .
$$

The first term on the right-hand side of ([6) is the "fermion part" of the total self-energy, while $\Pi_{M}^{\mu \nu}(p)$ represents the "Pauli-Villars part." We see that the $\mathcal{O}\left(b^{2}\right)$ contribution to $\Pi^{\mu \nu}(p)$ has a potential logarithmic divergence. However, if we were to improve the approximation (5) to obtain higher-order corrections in $b$, then the corresponding higher-order corrections to $\Pi^{\mu \nu}(p)$ will involve manifestly convergent integrals. Similarly, any $\mathcal{O}\left(b^{2}\right)$ terms involving positive powers of $p$ will also be power-counting finite. These finite terms must automatically obey the Ward identity, so we expect that by calculating the secondorder portion of $\Pi^{\mu \nu}(p=0)$, we should be isolating all the possible sources of Ward identity violation.

In fact, the $\mathcal{O}\left(b^{2}\right)$ contribution to the fermion part of the self-energy is

$$
\begin{aligned}
\Pi_{b^{2}}^{\mu \nu}(0)= & -i e^{2} \int \frac{d^{4} k}{(2 \pi)^{4}} \operatorname{tr}\left\{\gamma^{\mu} \frac{i}{\not k-m}\left(-i \not b \gamma_{5}\right) \frac{i}{\not k-m} \gamma^{\nu} \frac{i}{\not k-m}\left(-i \not b \gamma_{5}\right) \frac{i}{\not k-m}\right. \\
& {\left.\left[\gamma^{\mu} \frac{i}{\not k-m} \gamma^{\nu} \frac{i}{\not k-m}\left(-i \not b \gamma_{5}\right) \frac{i}{\not k-m}\left(-i \not b \gamma_{5}\right) \frac{i}{\not k-m}+(\mu \leftrightarrow \nu)\right]\right\} . } \\
= & i e^{2} \int \frac{d^{4} k}{(2 \pi)^{4}} \frac{1}{\left(k^{2}-m^{2}\right)^{4}} \operatorname{tr}\left\{\gamma^{\mu}(\not k+m) \not b(k-m) \gamma^{\nu}(\not k-m) \not b(k+m)\right. \\
& \left.+\left[\gamma^{\mu}(\not k+m) \gamma^{\nu}(\not k+m) \not b(k-m) \not k(k+m)+(\mu \leftrightarrow \nu)\right]\right\}
\end{aligned}
$$

The only Lorentz structures that may be present in $\Pi_{b^{2}}^{\mu \nu}(0)$ are $b^{\mu} b^{\nu}$ and $g^{\mu \nu} b^{2}$, so it is simplest to evaluate the self-energy by calculating $g_{\mu \nu} \Pi_{b^{2}}^{\mu \nu}(0)$ and $\frac{b_{\mu} b_{\nu}}{b^{2}} \Pi_{b^{2}}^{\mu \nu}(0)$. These calculations are fairly straightforward. For $g_{\mu \nu} \Pi_{b^{2}}^{\mu \nu}(0)$, we have

$g_{\mu \nu} \Pi_{b^{2}}^{\mu \nu}(0)=-i e^{2} \int \frac{d^{4} k}{(2 \pi)^{4}} \frac{\operatorname{tr}\left\{\left[2\left(\not \not \not k \not k-m^{2} \not b\right)+4(k-2 m)(\not k+m) \not b\right](k-m) \not b(k+m)\right\}}{\left(k^{2}-m^{2}\right)^{4}}$.

The $\mathcal{O}\left(k^{4}\right)$ terms in the numerator cancel under symmetric integration, and we are left with the finite integral

$$
\begin{aligned}
g_{\mu \nu} \Pi_{b^{2}}^{\mu \nu}(0) & =4 i e^{2} \int \frac{d^{4} k}{(2 \pi)^{4}} \frac{16 m^{2}\left[2(b \cdot k)^{2}-b^{2} k^{2}\right]-10 m^{4} b^{2}}{\left(k^{2}-m^{2}\right)^{4}} \\
& =-\frac{e^{2} b^{2}}{4 \pi^{2}} .
\end{aligned}
$$


Similarly, we also have

$$
\begin{aligned}
\frac{b_{\mu} b_{\nu}}{b^{2}} \Pi_{b^{2}}^{\mu \nu}(0) & =\frac{i e^{2}}{b^{2}} \int \frac{d^{4} k}{(2 \pi)^{4}} \frac{\operatorname{tr}\{\not \not(k+m) \not(3 \not k+m) \not(\not k-m) \not(\not k+m)\}}{\left(k^{2}-m^{2}\right)^{4}} \\
& =-4 i e^{2} \int \frac{d^{4} k}{(2 \pi)^{4}} \frac{2 m^{2} b^{2} k^{2}+m^{4} b^{2}}{\left(k^{2}-m^{2}\right)^{4}} \\
& =-\frac{e^{2} b^{2}}{8 \pi^{2}}
\end{aligned}
$$

where the logarithmic divergences again have canceled out.

From these results, it follows that

$$
\Pi_{b^{2}}^{\mu \nu}(0)=-\frac{e^{2}}{24 \pi^{2}}\left(2 b^{\mu} b^{\nu}+g^{\mu \nu} b^{2}\right) .
$$

This is the same result that we found previously in the $m=0$ case [19]. Since $p_{\mu}\left(2 b^{\mu} b^{\nu}+\right.$ $\left.g^{\mu \nu} b^{2}\right) \neq 0$, there is the potential for a violation of gauge invariance. However, since we are using a Pauli-Villars regularization, there is also a contribution from $\Pi_{M}^{\mu \nu}(p)$ that is second order in $b_{M}$. This contribution is precisely $\frac{e^{2}}{24 \pi^{2}}\left(2 b_{M}^{\mu} b_{M}^{\nu}+g^{\mu \nu} b_{M}^{2}\right)$. We are now in a position to place a natural condition on $b_{M}$; if transversality is to be maintained, the $\mathcal{O}\left(b^{2}\right)$ and $\mathcal{O}\left(b_{M}^{2}\right)$ contributions must cancel. This cancellation occurs exactly if $b_{M}= \pm b$. Therefore, we are left with a binary choice between two theories, characterized by different relative signs of $b$ and $b_{M}$, and these theories exhibit different values of the radiatively induced Chern-Simons coefficient. If $b_{M}=b$, then $\Delta k_{C S}$ vanishes, in accordance with earlier expectations. However, if $b_{M}=-b$, then

$$
\Delta k_{C S}^{\mu}=\frac{3 e^{2}}{8 \pi^{2}} b^{\mu}
$$

this is twice the value found in [10, 11, 14], since there are equal contributions from $b$ and $b_{M}$.

This is a novel situation. Unambiguously finite yet undetermined radiative corrections generally involve continuously variable parameters that describe the arbitrariness of the results [20, and indeed, in more general formulations of the theory we are discussing, there is a continuous ambiguity in the induced Chern-Simons term. However, in this instance, only a discrete set of values for $\Delta k_{C S}$ is allowed. Since zero is one of these allowed values, this formulation allows for the existence of a gauge-invariant Lagrange density, but it does not force the density to be so invariant. The Pauli-Villars formulation therefore allows the theory to possess a naturally vanishing induced Chern-Simons term without externally enforcing the gauge invariance of the Lagrange density. Moreover, since the Pauli-Villars regulator is covariant, it should be possible to generalize our calculations to occur in a weakly curved spacetime [21].

As we remarked in [19], the $\mathcal{O}(b)$ and $\mathcal{O}\left(b^{2}\right)$ terms in the photon self-energy both violate gauge invariance in the strongest fashions that are allowed by their tensor structures. 
The Chern-Simons term violates the gauge invariance of the Lagrange density; however, because the induced density necessarily involves the antisymmetric $\epsilon$-tensor, the Ward identity is preserved and the integrated action remains gauge invariant. $\Pi_{b^{2}}^{\mu \nu}(p)$ possesses no such special structure, and so it violates the Ward identity explicitly. In order to ensure gauge invariance, we have therefore been forced to place a very strong condition on the tensor structure of the second-order correction to the self-energy; we have insisted that it must vanish at $p=0$. Since there is more than one way to enforce this condition, we have found more than one possible value for $\Delta k_{C S}$. This is the origin of the discrete ambiguity in this formulation of the theory.

We may now ask what may happen if the mass scale $m^{2}$ is not large compared with the Lorentz-violation scale $\left|b^{2}\right|$. When the real fermions are massless, their $\mathcal{O}\left(b^{2}\right)$ contribution to $\Pi^{\mu \nu}(p)$ is exactly the $\Pi_{b^{2}}^{\mu \nu}(0)$ of equation (15). In this instance, as in the $\left|b^{2}\right| \ll m^{2}$ case, the use of a power series expansion to determine the second-order portion of the photon self-energy is justified, so the similarity of the results is somewhat unsurprising. If a Pauli-Villars regulator is used in this case, the Ward identity may be preserved, but the Chern-Simons term cannot be made to vanish. The choice is between $\Delta k_{C S}^{\mu}=\frac{e^{2}}{8 \pi^{2}} b^{\mu}$ and $\Delta k_{C S}^{\mu}=-\frac{e^{2}}{4 \pi^{2}} b^{\mu}$. So this formulation of the theory does not allow for $\Delta k_{C S}$ to vanish naturally if there are massless fermions coupled to the gauge field.

When a power series expansion in $b$ is not justified, the gauge noninvariant portions of the self-energy must be calculated directly from the nonperturbative propagator (31). Only if the result is of the form $A\left(b^{2}\right) b^{\mu} b^{\nu}+B\left(b^{2}\right) g^{\mu \nu} b^{2}$, with $A\left(b^{2}\right)=2 B\left(b^{2}\right) \leq 0$, will the Pauli-Villars regulator be capable of restoring the Ward identity. If we do choose to use a Pauli-Villars regulator and also insist upon gauge invariance as a requirement of our theory, then any breakdowns of the Ward identity when $\left|b^{2}\right| \nless m^{2}$ can be interpreted as giving restrictions on the allowed values of $b$. Applied to the standard model, this could imply that the scale of $b$-generated Lorentz violation should be smaller than the smallest nonzero fermionic mass scale present. However, this is all merely conjectural, since we do not know the general form taken by the self-energy when $\left|b^{2}\right| \nless m^{2}$, and because of the substantial additional complexity of the standard model.

This work has demonstrated that there exist new, unexpected subtleties in the structure of the radiatively-induced Chern-Simons term. We have identified new types of ambiguities in the value of the Chern-Simons coefficient; these ambiguities are derived from the use of a Pauli-Villars regulator and a nonperturbative formulation of the theory. If we do not insist that the Ward identity hold at second order, then the coefficient is completely arbitrary and is not even constrained to be parallel to $b$. However, if, more reasonably, we do insist on transversality of the vacuum polarization at higher orders, then there is a binary ambiguity in the value of the induced coefficient. If $m=0$, either of two nonzero values for $\Delta k_{C S}$ is allowed. However, if $\left|b^{2}\right| \ll m^{2}$, then $\Delta k_{C S}=0$ is allowed, in addition to a particular nonzero value. This may provide a satisfactory explanation for why the electromagnetic Chern-Simons coefficient vanishes in nature. 


\section{Acknowledgments}

The author is grateful to V. A. Kostelecký, M. Pérez-Victoria, and R. Jackiw for many helpful discussions. This work is supported in part by funds provided by the U. S. Department of Energy (D.O.E.) under cooperative research agreement DE-FG02-91ER40661.

\section{References}

[1] D. Colladay, V. A. Kostelecký, Phys. Rev. D 55, 6760 (1997).

[2] D. Colladay, V. A. Kostelecký, Phys. Rev. D 58, 116002 (1998).

[3] V. A. Kostelecký, R. Lehnert, Phys. Rev. D 63, 065008 (2001).

[4] R. Jackiw, S. Templeton, Phys. Rev. D 23, 2291 (1981).

[5] J. Schonfeld, Nucl. Phys. B 185, 157 (1981).

[6] S. M. Carroll, G. B. Field, R. Jackiw, Phys. Rev. D 41, 1231 (1990).

[7] S. M. Carroll, G. B. Field, Phys. Rev. Lett. 79, 2394 (1997).

[8] M. Goldhaber, V. Trimble, J. Astrophys. Astron. 17, 17 (1996).

[9] V. A. Kostelecký, C. D. Lane, A. G. M. Pickering, Phys. Rev. D 65, 056006 (2002).

[10] R. Jackiw, V. A. Kostelecký, Phys. Rev. Lett. 82, 3572 (1999).

[11] M. Pérez-Victoria, Phys. Rev. Lett. 83, 2518 (1999).

[12] M. Pérez-Victoria, JHEP 04, 032 (2001).

[13] S. Coleman, S. L. Glashow, Phys. Rev. D 59, 116008 (1999).

[14] J. M. Chung, Phys. Lett. B 461, 138 (1999).

[15] J. M. Chung, P. Oh, Phys. Rev. D 60, 067702 (1999).

[16] W. F. Chen, Phys. Rev. D 60, 085007 (1999).

[17] J. M. Chung, Phys. Rev. D 60, 127901 (1999).

[18] A. A. Andrianov, P. Giacconi, R. Soldati, JHEP 02, 030 (2002).

[19] B. Altschul, Phys. Rev. D 69, 125009 (2004).

[20] R. Jackiw, Int. J. Mod. Phys. B 14, 2011 (2000).

[21] V. A. Kostelecký, Phys. Rev. D 69105009 (2004). 